\title{
Mechanism of hydration of urea and guanidium ion: A model study of denaturation of proteins
}

\author{
Fateh S. Nandel, Rajnish Verma, Balvinder Singh and Dharam V.S. Jain ${ }^{a}$ \\ Department of Biophysics, Department of Chemistrya ${ }^{\text {, }}$ Panjab University, \\ Chandigarh-160 014. India.
}

\begin{abstract}
The hydration of urea and guanidium ion have been studied by quantum chemical and molecular mechanics methods. The hydration of urea takes place only through the amino groups and not through the carbonyl group. One mole of urea decreases the free concentration of water by seven moles and one mole of guanidium ion by twelve moles. The interaction energies $\left(\Delta \mathrm{E}^{\prime} s\right)$ for the hydration of urea and guanidium ion in both the primary and secondary spheres are more than that of waterwater interactions. Also, the interaction energy of urea and guanidium ion with water is more than the interaction energy of urea and guanidium ion with the potential hydrogen bonding sites in the peptide groups of the model pentapeptides. The "collagen type structure" (with $\phi_{\mathrm{i}}=-30^{\circ}, \psi_{\mathrm{i}}=120^{\circ}$ ) has been taken as model for functional domain which is stabilized by interaction with water through free potential hydrogen bonding sites. It is shown that these denaturants, reduce the free concentration/activity of water effectively and remove the water molecules from the protein surface. The computational results also demonstrate why lower concentrations of guanidium ion is sufficient to bring about the denaturation in comparison to urea. The interaction studies also reveal that there is no complex formation between the peptides groups and denaturant molecules. The cause of protein aggregation in the presence of denaturants and hence, the loss of biological activity is also discussed.

Key words: Urea, Guanidium ion, Hydration, Protein, Denaturation/Aggregation.
\end{abstract}

\section{INTRODUCTION}

The denaturation of proteins/enzymes occurs due to the environmental changes such as temperature, $\mathrm{pH}$, pressure and addition of denaturants like urea and guanidium ( $\mathrm{Gu}$ ) hydrogen chloride (ref. 1- 3). The universal destabilizing action of urea is well known (ref. 4). Urea enhances the solubility of nonpolar compounds in water, reduces the tendency of the surfactants to form micelles and destabilizes phospholipid membrane structure. All these effects can be accounted for on a semi-quantitative basis by the influence of urea on the intermolecular hydrogen bonded structure of liquid water. Solvent additives can affect macromolecular structure by direct interaction with the macromolecule or by indirect action through effects on the structure and properties of the solvent or by a combination of both these mechanisms (ref. 5). However, there is little understanding about the details and roles of such interactions.

The effect of urea and guanidium ion on the intermolecular hydrogen bonded structure of water has been studied. It will also be interesting to examine whether the hydration of urea originates from the carbonyl or amino group or from both the groups. The guanidium ion differs from urea molecule as it contains $-\mathrm{N}-\mathrm{H}$ moiety in place of $>\mathrm{C}=\mathrm{O}$. Therefore, hydration of this molecule may reveal why lower 
concentration of guanidium ion is sufficient in comparison to urea in the protein/enzyme denaturation. Further, the interaction of water, urea and guanidium ion with the potential hydrogen bonding sites of the model peptide backbone in both the structural and functional domains may unravel the mechanism of denaturation.

\section{METHODS OF CALCULATIONS}

The geometries of water, urea, guanidium ion \& the peptides (corresponding to both structural and functional domains) and their supermolecules have been optimized using the potential function of the type as suggested by Vinter et al. (ref. 6).

The interaction energies of water with urea, with guanidium ion $\&$ with peptide backbone (i.e. with the $>\mathrm{C}=\mathrm{O}$ and $\mathrm{N}-\mathrm{H}$ groups) and of urea \& guanidium ion with the peptide groups have been calculated by GRINDOL (Ghost and Rydberg Intermediate Neglect of Differential Overlap) method (ref. 7). This semi-empirical all valence method has been successfully employed for the calculation of ground and excited state properties of isolated molecules and the intermolecular interactions. In this method, the total energy for a closed shell system is given by the expression (ref. 7):

$$
\begin{aligned}
& E_{\text {total }}=0.5 \sum_{\mu} \sum_{\nu} P_{\mu v}\left(H_{\mu \nu}^{c o r e}+F_{\mu v}\right)+\sum_{A>B} \sum_{A B} E_{A B}^{c o r e} \\
& \text { where } E_{A B}^{c o r e} \text { denotes repulsion energy of the atomic cores } A \text { and } B .
\end{aligned}
$$

Interaction energy, $\Delta \mathrm{E}$, between the molecules $\mathrm{X}$ and $\mathrm{Y}$ is calculated by using SuperMolecule (SM) approach

$$
\Delta \mathrm{E}_{\mathrm{SM}}=\mathrm{E}_{\mathrm{XY}}-\mathrm{E}_{\mathrm{X}}-\mathrm{E}_{\mathrm{Y}}
$$

where $E_{X}, E_{Y}$ and $E_{X Y}$ denote the SCF energies of the molecules $X, Y$ and Supermolecule $\mathrm{XY}$, respectively.

The hydration of urea and guanidium ion have been studied by stepwise addition of water at the interacting sites by taking into account all possible geometries in both the primary and secondary hydration spheres. This has been followed by optimization and calculation of the interaction energy. The most stable configuration has been chosen as starting geometry for the subsequent interaction of water molecules. Since, these denaturants influence the structure of water, therefore, it becomes imperative to study water-water interactions also. Similar procedure has been followed for the interaction of water, urea and guanidium ion with the potential binding sites of the model peptides Ac$(\mathrm{Ala})_{\mathfrak{n}}$-NHMe containing one to four alanine residues only, in the required conformation corresponding to both structural (with $\phi_{\mathrm{i}}=-30^{\circ}, \psi_{\mathrm{i}}=-60^{\circ}$ ) and functional (with $\phi_{\mathrm{i}}=-30^{\circ}, \psi_{\mathrm{i}}=120^{\circ}$ ) domains (ref. 8).

\section{RESULTS AND DISCUSSION}

\section{Interaction of Urea and Guanidium ion with Water:}

Two schemes have been taken into consideration for the hydration of urea. In the first scheme, water molecule with one of its hydrogen approaches the carbonyl group of urea; while in the other, water molecule with its oxygen approaches the hydrogen of amino group in urea at two distinct sites. When the water molecule is made to interact with carbonyl group of urea, no stabilization is obtained, while the interaction of water vith amino $\left(-\mathrm{NH}_{2}\right)$ group leads to net stabilization, suggesting that the hydration starts from the amino group of the urea. 
To explain why the hydration of urea does not start from the carbonyl group, the electronic charges on solated molecules i.e. water, urea \& $\mathrm{N}$-methyl acetamide and urea in the presence of four water

TABLE 1. Charges on some atoms in water, urea and $\mathrm{N}$-methylacetamide.

\begin{tabular}{lllll}
\hline & \multicolumn{4}{c}{ Atomic Centres } \\
\cline { 2 - 6 } Compounds & $\mathrm{C}$ & $\mathrm{N}$ & $\mathrm{O}$ & $\mathrm{H}$ \\
\hline Water & - & - & -0.44 & 0.22 \\
Urea & 0.73 & -0.38 & -0.70 & - \\
Urea+4H $2 \mathrm{O}$ & 0.82 & -0.07 & -0.74 & - \\
N-Methylacetamide & 0.07 & -0.41 & -0.60 & - \\
& & & & \\
\hline
\end{tabular}
molecules in primary hydration sphere have been calculated (Table 1). The results show that the carbonyl carbon carries $0.73 \mathrm{e}$ and $0.82 \mathrm{e}$ positive charge in urea and hydrated urea, respectively. This leads to repulsion between the hydrogen atom of the approaching water molecule carrying partial positive charge. Further, the calculations show that in Nmethyl acetamide (a model representative of peptide bond), the carbonyl carbon and nitrogen of amino group carries $+0.07 \mathrm{e}$ and $-0.41 \mathrm{e}$ charge, respectively. The magnitude of charges on carbonyl carbon is much less as compared to that on the carbonyl carbon of the urea molecule. This clearly indicates that water can interact with both the moieties (i.e. amino and carbonyl groups) of peptide bonds and the magnitude of charges also suggest that the interaction with carbonyl oxygen will be more than that with the amino nitrogen. This fact is supported by crystallographic data on thirteen well defined proteins in which the internal water molecules form 1.9 times more hydrogen bonds to the main chain carbonyls than to the main chain nitrogen (ref. 9).

The results for the hydration of urea by gradual addition of water molecules corresponding to the most stable geometry are summarized in Fig. 1. In the primary hydration sphere of urea, four water molecules are involved while three water molecules are accommodated in its secondary hydration sphere. The interaction of water molecules through its oxygen with any of the $-\mathrm{N}-\mathrm{H}$ groups of the guanidium ion gives almost the same interaction energy. It is found that six water molecules are accommodated in each of the primary hydration sphere as well as in the secondary hydration sphere, thereby, involving a total of twelve molecules of water in the hydration of guanidium ion (Fig. 1). The interaction energies at each step of hydration are greater than the water-peptide and water-water interactions for both the urea and guanidium ion. Thus, one mole each of urea and of guanidium ion takes care of seven and twelve moles of water, respectively and decrease the free concentration/activity of water, effectively. The decrease in activity of water as a function of denaturant concentration may be interpreted as decrease in hydrophobic interactions between water and the apolar molecules. This viewpoint also finds support from the experimental studies by Schellman (1990) (ref. 10), Thayer et al. (1993) (ref. 11) and Sijpkes et al., (1993) (ref. 12). As the concentration of denaturants is increased, more of the water molecules get associated with the denaturants and this is usually referred to as the structure breaking effect of these denaturants (ref. 4) and results in creation of cavities of varying sizes in the bulk water structure in conformity with findings of earlier workers (ref. 13-15). Thus, both the effects (decrease in water activity and creation of cavities) taken into account, provide an acceptable explanation for the increasing solubility of the protein or apolar molecule in the solutions of urea or guanidium ion.

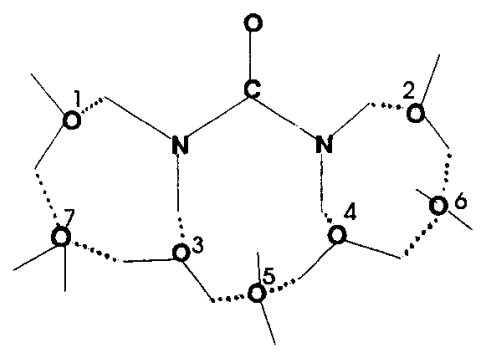

$(-402.1)$

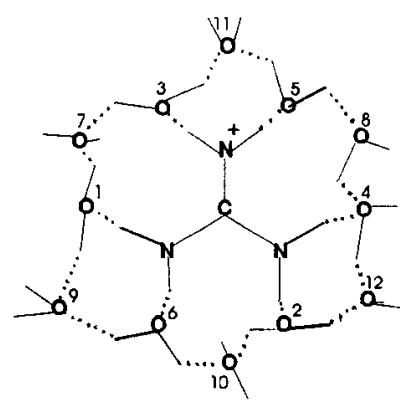

$(-543.5)$

Fig. 1 The hydration of urea and guanidium ion: The order of stepwise addition of water molecules going in for the most stable configuration is indicated by Arabic numeral. Interaction energies in $\mathrm{kJ} / \mathrm{mol}$ are given in parenthesis. 


\section{Interaction of Water, Urea and Guanidium ion with the Peptide groups in different Conformational states:}

Usually, the peptides/proteins are studied in the aqueous media to mimic the native environment of the biomolecule. To understand the interactions of water, urea and guanidium ion with the potential hydrogen bonding sites in the peptide bond, the computational results for the model pentapeptides- Ac$\mathrm{Ala}_{4}-\mathrm{NHMe}$ and $\mathrm{Ac}-\mathrm{Ala}_{4}-\mathrm{OMe}$ in the desired conformation are discussed.

The functional domains are usually found in the loops or turns and/or at antigenic or metal binding surfaces and the $\phi, \psi$ values for this "collagen type structure" on the basis of computational results (ref. 8,16 ), database analysis (ref. 17), spectroscopic CD (ref. 18-21) and IR results (ref. 22) corresponds to $\phi_{\mathrm{i}}=-30^{\circ}, \quad \psi_{\mathrm{i}}=120^{\circ}$. In this conformational state, the potential hydrogen bonding sites are free (Fig. 2) and this structure is energetically favoured over the helices for short peptide sequences i.e. maximally upto five amino acid residues, with no intramolecular hydrogen bond interactions and as the number of amino acid residues increase, the propensity of the "collagen type structure" decreases (ref. 23). This structure is stabilized by interaction of water with the peptide linkages accompanied by a small change in the $\phi, \psi$ values of the peptide backbone which is consistent with the X-ray crystal data (ref. 24) that this structure is found on the surfaces of the

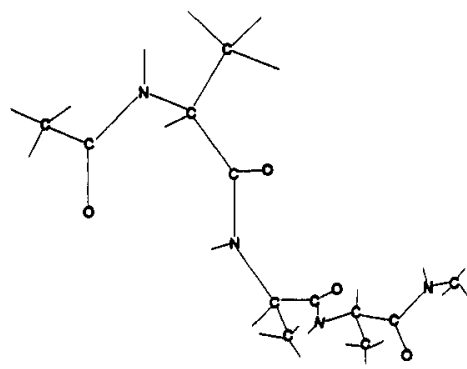

Fig. 2 A graphical view of Ac-Ala $-\mathrm{NHMe}$ in "collagen type conformation" with $\phi_{i}=30^{\circ}$, $\psi_{i}=120^{\circ}$ showing that all the potential hydrogen bonding sites are free for intermolecular interactions. proteins. The interaction energies of urea and guanidium ion with carbonyl and amino groups of the peptide backbone are much less than the interaction of water with these denaturants, respectively. Hence, these denaturants have hardly any preference for binding to the peptide groups.

The structural domains of globular proteins are composed of helices $(\alpha$ or 310 ) and/or $\beta$-sheets (ref. 25,26 ). Water is present in the structural domains as discrete single molecules and also in clusters. In actidine, comprising of 220 amino acid residues, crystal structure contains many clusters of water molecules but only a few of these molecules make direct contact with the protein molecule (ref. 9,27), additional contacts are via water bridges. The water molecules acting as bridges between the terminal potential hydrogen bonding sites and between the chains of protein interior act as stabilizer (ref. 28-38). It may also be pointed out that the internal water molecules are highly conserved in plant thiol proteases- papain (ref. 39,40) and actidine from different sources (ref. 9) and in several serine proteases such as chymotrypsin (ref. 41), trypsin (ref. 42-44), elastase (ref. 45) \& kallikrein (ref. 46). Therefore, the interaction study of water with the free potential hydrogen bonding sites by taking model peptide in $3_{10}$ helical secondary structure with $\phi_{\mathrm{i}}=-30^{\circ}, \psi_{\mathrm{i}}=-60^{\circ}$ has been carried out. The helix with these $\phi, \psi$ values is the most stable one in the shorter peptides with less than ten amino acid residues (ref. 16; PCILO results- ref. 47,48).

The magnitude of interaction energies of the denaturants with peptide groups in both the functional and structural domains are comparable to that of water-water interaction and water-peptide interactions $(-39.75 \pm 1.25 \mathrm{~kJ} / \mathrm{mol})$ but are much less than the interaction energies between the denaturants and water. This clearly rules out the possibility of a complex formation between the peptide and denaturant molecule. Recent experimental studies by Scholtz et al. (1995) on urea unfolding of peptide helices (ref. 49) and by Sijpkes et al., (1993) on the interaction of diketopiperazine with urea in aqueous solution (ref. 12) also lend support to the above findings. A very small value of the association constant has been reported by these workers for the complex between the urea- diketopiperazine and 
urea-peptide. Further, very recent microcalorimetric studies by Loh et al., have ruled out the complex formation between urea and amino acids (ref. 15), thus, confirms the computational findings. Hence, it may not be logical to think whether the denaturation starts from the $\mathrm{N}$ - or $\mathrm{C}$ - terminal,

Therefore, it is reasonable to state that the denaturant molecules firstly, decrease the activity of water molecules, creating cavities in water structure and then, removes the water molecules from the protein. It is also worth pointing out that the interaction energy of both the denaturants with water is much less than the energy of bond formation for disulphide linkage (ref. 50) and thus, the secondary structure will not open up completely in the proteins containing disulphide linkages. The removal of water from the functional domain results in aggregation of protein molecules by making intramolecular hydrogen bonds and hence, the loss of biological activity. The same explanation equally holds true for the denaturation of proteins at low and high temperatures in aqueous solutions (ref. 51- 54). It is the water which freezes out as ice on lowering of temperature and not the protein. Thus, the water is removed from the functional domain which renders the potential hydrogen bonding sites to be free leading to the aggregation of the protein and the loss of biological activity. At higher temperature, the hydrogen bonds between the peptide and water molecules break and the water is removed from the active sites, leading to the loss of biological activity as discussed above.

\section{Acknowledgment}

We are thankful to Dr. J.Lipinski, Institute of Organic and Physical Chemistry, Technical University of Wroclaw, Wroclaw, Poland for the GRINDOL program.

\section{REFERENCES}

1. C. Tanford. Adv. Pro. Chem. 23, 121 (1968).

2. C. Tanford. Adv. Pro. Chem. 24, 1 (1970).

3. D.B. Wetlaufer, S.K. Malikk, L. Stoller and R.L. Coffin. J. Am. Chem. Soc. 86, 508 (1964).

4. T.E. Creighton. Proteins, Freeman, New York (1984).

5. K. Gekko and S.N. Timasheff. Biochem. 20, 4667 (1981).

6. J.G. Vinter, A. Davis and M.R. Suders. J. Comput. Aided Molec. Des. 1, 31 (1987).

7. J. Lipinski. Int. J. Quan. Chem. 26, 423 (1988).

8. F.S. Nandel and A. Ahluwalia. Int. J. Quant. Chem. 55, 61 (1995).

9. E.N. Baker and R.E. Hubbord. Prog. Biophys. Mol. Biol. 44, 97 (1984).

10. J.A. Schellman. Biophys. Chem. 37, 121 (1990).

11. M.M. Thayer, R.C. Haltiwanger, V.S. Allured, S.C. Gill and S.J. Gill. Biophys. Chem. 46, 165 (1993).

12. A.H. Sijpkes. G.J. van de Kleut and S.C. Gill. Biophys. Chem. 46, 171 (1993).

13. N. Muller. Acc. Chem. Res. 23, 23 (1990).

14. N. Muller. J. Phys. Chem. 94, 3856 (1990).

15. W. Loh, A.E. Beezer and J.C. Mitchell. Thermochim. Acta 255, 83 (1995).

16. Amandeep. M.Sc. thesis, Panjab University, Chandigarh, India (1993).

17. A.A. Adzubei, F. Eisenmenger, V.G. Tumanyan, M. Zinke and N.G.Esipova. Biophys. Biochem. Res. Commun. 146, 934 (1987).

18. N. Greenfield and G.D. Fasman. Biochem. 8, 4108 (1969).

19. G. Vertuani, C. Falcomer, M. Boggian, G. Pochetti, C. Cerrini, M. Ricci and A. Scatturin. Int. J. Pept. Pro. Res. 33, 162 (1989).

20. G. Siligardi, Ph.D. Thesis, University of London, London (1990).

21. G. Siligardi, A.A. Drake, P. Mascagni, D. Rowland and F. Brown. Eur. J. Biochem. 199, 545 (1991).

22. M. Jackson, P.I. Harris and D. Chapman. Biophys. Biochem. Acta 998, 75(1989). 
23. F.S. Nandel, B. Singh and N. Malik (Unpublished results).

24. A.A. Adzubei and J.E. Sternberg. J. Mol. Biol. 229, 472 (1993).

25. L. Pauling, R.B. Corey and H.R. Branson. Proc. Natl. Acad. Sci. (USA) 37, 205 (1951).

26. L. Pauling and R.B. Corey. Proc. Natl. Acad. Sci. (USA) 37, 729 (1951).

27. E.N. Baker. J. Mol. Biol. 141, 441 (1980).

28. C.C.F. Blake, W.C.A. Pulford and P.J. Artymiuk. J. Mol. Biol. 167, 693 (1983).

29. M.M. Teeter. Proc. Natl. Acad. Sci. (USA) 81, 6014 (1984).

30. E.N. Baker, T.L. Blundell, J.F. Cutfield, S.M. Cutfield and E.J. Dodson. Proc. Trans. R. Soc. London Ser B 319, 369 (1988).

31. D.C. Rees, M. Lewis and W.N. Lipscomb. J. Mol. Biol. 168, 369 (1983).

32. A. Wlodawer, J. Deisenhofer and R. Huber. J. Mol. Biol. 193, 145 (1987).

33. X. Cheng and B.P. Schoenborn. Acta Crystallogra. Sect. B46, 195 (1990).

34. M.S. Lehman and G. Zaccai. Biochem. 23, 1939 (1984).

35. K. Takahashi, A. Ajima, T. Yoshimoto and Y. Inada. Biophys. Biochem. Res. Commun. 125, 761 (1984).

36. R.M. Guinn, H.W. Blanch and D.S. Clark. Enzyme Microb. Technol. 13, 320 (1991).

37. B.D. Blasio, V.D. Duca, A. Lombardi, C. Pedone, G. P. Lorenzi and E. Benedetti. Int. J. Pept. Pro. Res. 45, 100 (1995).

38. S. Cerrini, D.Lamba. A scattrurin and G. Ughetto. Biopolymers 28, 409.(1989).

39. M.M. Teeter. In Protein Folding (L.M. Gierasch \& J. King, eds.), Americam Association for the Advancement of Science, p. 44. Washington, D.C. (1990).

40. J.M.C. Berendsen. In Water- A Comprehensive Treatise Vol 5, (F. Franks ed.), Plenum, New York (1975).

41. J.J. Birktoft and D.M. Blow. J. Mol. Biol. 68, 187 (1972).

42. W. Bode and R. Huber. In Molecular and cellular Basis of digestion, (P. Desnuelle, H. Sjostrom, O. Noren eds.) pp.187 New York: Elsevier (1986).

43. J.L. Chambers and R.M. Stroud. Acta Crystallogr. Sect. B35, 186 (1979).

44. H. Bartmirk, L. Summers and H. Bartsch. J. Mol. Biol. 210, 813 (1989).

45. L. Sawyer, D.M. Shotton, J.W. Campbell, P.L. Wendel, H. Muirhead et al. J. Mol. Biol. 118, 137 (1978).

46. W. Bode, Z. Chen, K.S. Bartels, C. Kutzbach, G. Schmidt- Kastner et al. J. Mol. Biol. 164, 237 (1983).

47. B. Pullman. In Quantum Mechanics of Molecular Conformations (B. Pullman ed.) p. 295. Wiley, New York (1976).

48. B. Pullman and A. Pullman. Adv. Pro. Chem. 28, 347 (1974).

49. J.M. Scholtz, D. Barrick, E.J. York, J.M. Stewart and R.L. Baldwin. Proc. Natl. Acad. Sci. (USA) 92, 185 (1995).

50. L.N. Ferguson. In The Modern Structural Theory of organic chemistry. p. 48. Prentice-Hall of India Pvt. Ltd., India (1963).

51. F. Franks and J.E. Desnoyers. Water Sci. Rev. 1, 171 (1985).

52. D.W. Davidson. In Water-A Comprehensive Treatise Vol. 6, (F. Franks ed.), Plenum, New York (1979).

53. D. Eagland. In Water -A Comprehensive Treatise Vol. 4, (F. Franks ed.), p.305 Plenum, New York, (1975).

54. F. Franks. In Polysaccharides in foods (J.M.V. Blanshord and J.R. Mitchel, eds.), p. 33. Butterworths, London (1979). 